\title{
Using a Mobile Robot with Interpolation and Extrapolation Method for Chemical Source Localization in Dynamic Advection-Diffusion Environment
}

\author{
Xiang Gao, Levent Acar \\ Department of Electrical and Computer Engineering, Missouri University of Science and Technology, USA
}

\begin{abstract}
Article Info
Article history:

Received Feb 8, 2016

Revised Apr 24, 2016

Accepted May 16, 2016

\section{Keyword:}

Chemical Source Localization

Computational Method

Fluid Flow Systems

Mobile Robot

Sensors Network

ABSTRACT

This paper address the problem of mapping likely particle path derived from a chemical source using interpolation and extrapolation method. Order localization is the problem of finding the source of an odor or other volatile chemical. Most localization method require the robot to follow the odor plume along its entire length, which is time consuming and may be especially difficult in a cluttered environment. In this paper, a map of sensors' environment was used, together with the path line of airflow, to predict the pattern of air movement. The robot then used the airflow pattern to reason about the probable location of the odor source. This demonstrates that interpolation and extrapolation method can be used to assist odor localization search and indicates that similar techniques have great operating in an unstructured environment to reason about its surroundings. This paper present details of getting the model of particle path using interpolation and extrapolation method, model of particle path surrounding the obstacles and openings, result of practical odor source location simulation.
\end{abstract}

Copyright () 2016 Institute of Advanced Engineering and Science. All rights reserved.

\section{Corresponding Author:}

Xiang Gao,

Departement of Electrical and Computer Engineering,

Missouri University of Science and Technology,

301 W. $16^{\text {th }}$ St, Rolla, MO, USA.

Email:xghnc@mst.edu

\section{INTRODUCTION}

The detection of the airborne chemicals presents a different type of challenge than the more traditional detection efforts, such as visual-based detection or propagating signal detection [1, 2, 3, 4, and 5]. The chemicals that are airborne tend to drift in various directions due to wind, up-draft, and obstacles. As a result, isolation of the source of such particles becomes considerable difficult and dependent on topography and environment.

There has been some previous research on the detection and modeling of airborne particles, plume location and tracking. However, most of such research is based on sensor information on moving robots that are guided by the detectors. These types of sensing robots are assumed to move about freely following the trail of a chemical signature, while they're continuously sensing for the particles. Both of these assumptions are not valid in inaccessible and hostile environments with sensors that can either function once or need along rejuvenation time cycles.

In our approach to the problem of chemical particle detection and source location, we use a small number of chemical sensors that are sparsely scattered around an area only known by a two-dimensional map. In real-world problems, we anticipate that a mobile robot would drop some of these sensors on the area of interest while taking some areal pictures. We assume that the sensor data along with the map are transmitted to a nearby location perhaps to a vehicle that will be traveling through the area of interest. We would like to use the maximum available information content to generate first a model of the chemical 
particle distribution, and then locate the source of the particles based on the model. By the reasoning system, we teach the mobile robot to move into the area where most-likely hood of chemical source are.

\section{DESCRIPTION OF THE METHODOLOGY}

As a test case, we consider a three sensor configuration system as in Figure 1. In the figure, the thick black lines are the boundaries of the room, the red dots are the sensor locations, and the red dotted lines designate the border of the boundary zone.

Some chemical sensors are designed to detect simply the existence of a chemical particle and trigger a positive result when the concentration amounts are above a preset threshold level. In our design, instead of the threshold, we make use of the actual concentration levels that are detected. This approach along with some other data enables us to model the flow of the particles and the location of the source. Each sensor provides the co-located sensory information of the wind velocity, the concentration of the particles, and the concentration differential preferably perpendicular to the wind direction. The concentration differential information is obtained not by an additional sensory device but by an off-centered multi-orifice detection hardware configuration. In our derivations, we assume that the differential information is perpendicular to the wind direction, but we can accommodate any non-zero known angular orientation simply by a coordinate transformation. Designating the location of the sensors by $(\mathrm{x}, \mathrm{y})$, we represent the flow of air by $\delta x, \delta y$. Similarly, we represent the sensed particle concentration by s and the concentration gradient by $\delta s$.

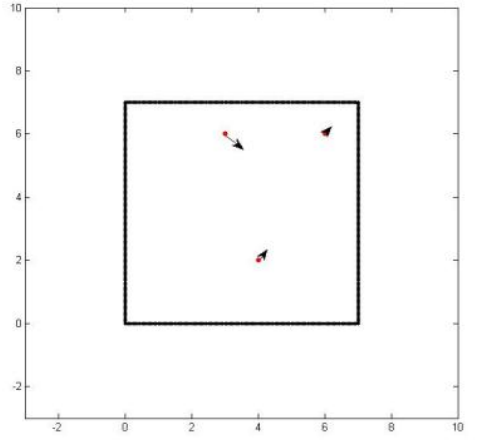

Figure 1. The location of three sensors in a square enclosure.

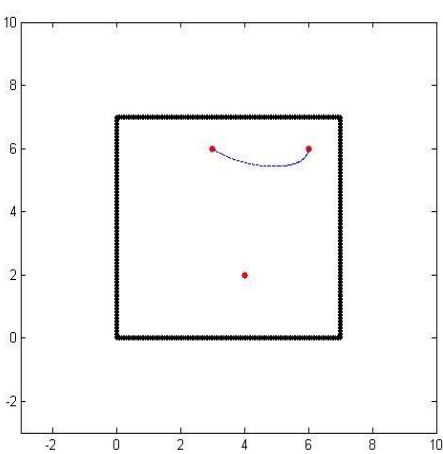

Figure 2. An air-borne particle path with matching terminal velocities.

Once we obtain the sensory information, we start with an approximation of the particle path. In order to avoid multiple solutions, we make a number of assumptions.

Assumption 1: The air-borne particles travel the most direct route.

Based on Assumption 1, we configure paths that go through the sensor locations, such that the paths satisfy the locations as well as the differentials. This approach leads to a parametric cubic-polynomial representation of the path in terms of a variable t. We use the cubic Hermite splines with the end point differentials weighted three times, such that

$$
\left.x(t)=(2(x(0)-x(1))+3(\delta x(0)+\delta x(1))) t^{3}+3(x(1)-x(0))-3(\delta x(1)+2 \delta x(0))\right) t^{2}+3 \delta x(0) t+x(0),
$$$$
\left.y(t)=(2(y(0)-y(1))+3(\delta y(0)+\delta y(1))) t^{3}+3(y(1)-y(0))-3(\delta y(1)+2 \delta y(0))\right) t^{2}+3 \delta y(0) t+y(0) .
$$

where the parametric curve starts at one sensor location at $\mathrm{x}(0), \mathrm{y}(0)$ and ends at the other sensor location at $\mathrm{x}(1), \mathrm{y}(1)$ as t goes from 0 to 1 . Figure 2 shows the spline approximation of a particle path from one sensor to another with matching initial and final velocities.

If we strictly apply this interpolation method, we end up two possible choices, one path going from Sensor 1 to Sensor 2 and another path going from Sensor 2 to Sensor 1. We could explore both possibilities or have a decision making process based on other factors to eliminate one of the choices. Here, as a first step, we choose the shortest path option.

Assumption 2: The path of the air-borne particles has minimal length.

Even though, we now have a path from one sensor to another, there are still couple of issues to be resolved. The first issue is related to the underlying presumption that a particle somehow would travel from one sensor to the other even though the sensors are at arbitrary locations. To correct this problem, we rely on 
the dissipation property of the particles. We compute the expected concentration value along the computed path and compare it with the actual sensed concentrate on value. Based on the error and the measured gradient concentration, we determine a new location perpendicular to the initial path where the expected and sensed concentration values match. We then compute the corrected path going through one of the sensors and the new location. When we repeat the process forwards from one sensor and backwards from another one, we end up getting two consistent paths with correct concentration values. Figure 3 shows the two paths generated by matching the expected and sensed concentration values.

The second issue is related to the choice of the parameter $t$. In our parametrization, we chose t to start at 0 at one of the sensors and end at 0 initially at another sensor at 1 . We would like to have the parameter be a good representative of actual travel time, since we also would like to obtain connected paths. To correct this problem, we compute the speed at every point along the path as a linear function of the distance from one sensor to the other one while matching the sensed speed values at the two end points. Figure 4 shows the two paths with equally-timed distances.

In the existence of multiple sensors, we need to make sure that the paths continue smoothly passing through the neighborhood of sensors. As an example, if we generate a path from Sensor 1 towards Sensor 2, then we need to make sure that at the terminal point near Sensor 2 we have another path towards Sensor 3. In this example, this extended path goes through Sensor 1 and the neighborhoods of Sensors 2 and 3 due to path updates for the consistency of the particle propagation. To obtain a general development of all possible paths passing through the neighborhoods of sensors, we introduced a couple of more assumptions.

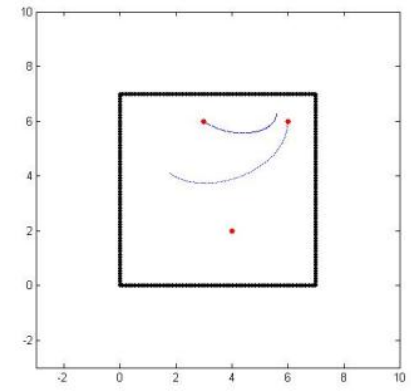

Figure 3. Consistent air-borne particle paths between two sensors

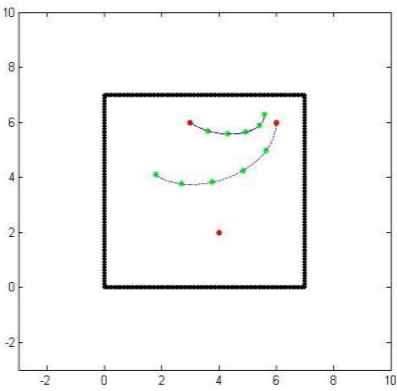

Figure 4. Consistent air-borne particle paths between two sensors

Assumption 3: The individual segments of the paths that span multiple sensor regions have to have the same flow directions.

Assumption 4: The paths that span multiple sensor regions always go through at least one sensor.

Based on the above assumptions, Figure 5 shows the three curves that go through the three sensor regions of Figure 1, and Figure 6 shows the same paths with equally-timed distances.

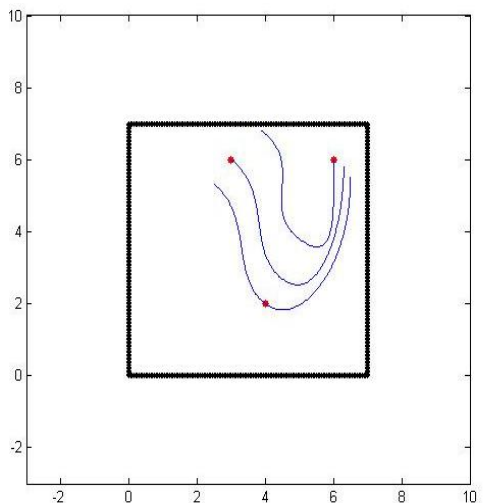

Figure 5. Consistent air-borne particle paths among three sensors

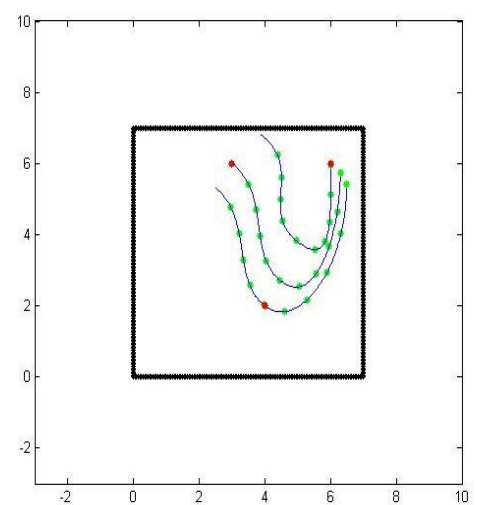

Figure 6. Consistent air-borne particle paths among three sensors 
As we observe from the Figures 4 and 6, there are two set of possible particle flow directions. At this point, we pursue both possibilities and make a decision on the actual flow path later by a conflict resolution layer.

As we described above, in the process of determining the particle paths that go through the sensors, we primarily rely on the sensory values, the particle dissipation properties, and interpolation. However, in order to generate a full coverage of paths for the whole room, we need to extrapolate as well.

In the first step of the interpolation, we extend the primary paths that go through the sensors with linear approximations of the air flow parameters, and the particle dissipation properties of the particle concentrations. Figures 7 and 8 show the path extensions, where we purposely extended the paths beyond the room boundaries. We will take care of the portions that go beyond the boundaries specially after the whole room coverage below.

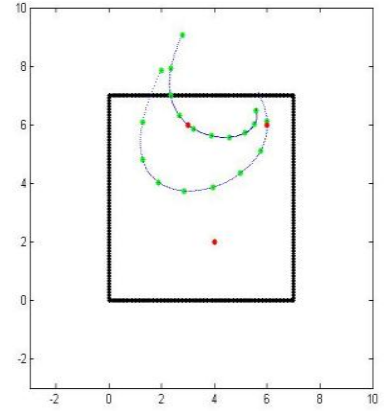

Figure 7. Primary air-borne particle path extensions going through two sensors

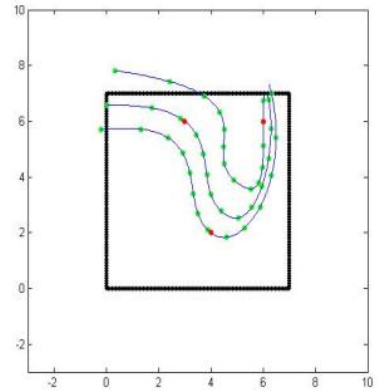

Figure 8. Primary air-borne particle path extensions going through three sensors

In the next step of the extrapolation, we fill the whole room with secondary paths. For the secondary paths that are between two adjacent primary paths, we determine the normals (perpendicular lines to the tangents of the paths), and use the intersection points of the normals to generate a secondary path. We assign the average values of the particle concentrations and the concentration gradients on these paths. For the secondary paths that are on the outside regions of the primary paths, we use similar normal extensions, but we extrapolate the particle concentrations and the concentration gradients. Figures $9 \& 10$ show the path extensions as well as the whole room coverage with primary and the secondary paths.

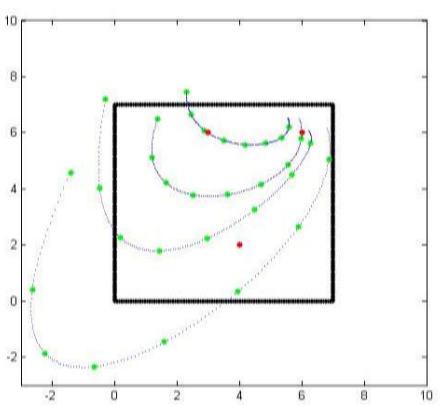

Figure 9. Primary and secondary air-borne particle paths going through two sensors

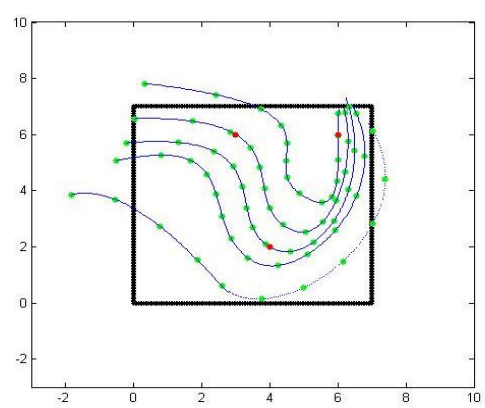

Figure 10. Primary and secondary air-borne particle paths going through three sensor

He extended primary and the secondary paths depend on the best data fit based on the sensory data. In the generation of these paths, w e excluded the effects of the room boundaries. Obviously, if we truncate the paths at the boundary, we'll generate unnatural particle behavior, where particle would originate on one side of the boundary and disappear on the other side. To model the particle path along these types of boundary structures, we need to incorporate realistic flows of air in the neighborhood of the boundaries. Our approach to handle the boundaries is based on a convex buffer zone in the neighborhood of the room boundary, where the air-borne particle flow is distorted in the direction of the boundary. In this zone, we map 
the portions of the paths, which are in the buffer zone or outside the room boundary, in a manner that preserves the continuity of the location and the derivative of a particle entering this zone. infinitely.

Assumption 5: The paths that go across the boundary regions always go close to the boundary

If we denote the perpendicular distance of a point on one of the paths by $d$, then we compute the new distance $\hat{d}$ as $\hat{d}=w \tanh (d / w)$, where $\mathrm{w}$ is the width of the buffer zone. This approach maps the paths at infinity onto the room boundary and enables all of the portions outside the room boundary to be in the buffer zone. Figures 11 and 12 show the complete set of paths confined within the room boundary.

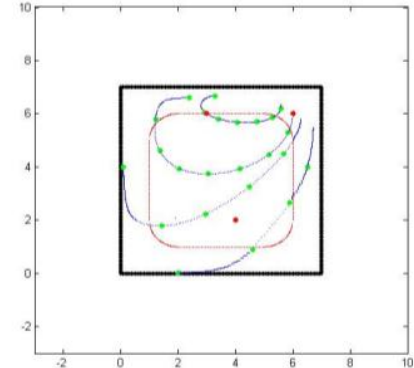

Figure 11. Boundary confined air-borne particle paths going through two sensors

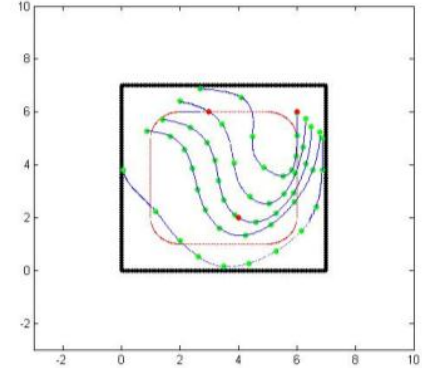

Figure 12. Boundary confined air-borne particle paths going through three sensors

To further validate our approach, we applied the same principles on a slightly larger room in Figure 13 and with twice the air speed in Figure 14. As we can observe from these figures, our approach provides satisfactory and consistent air-borne particle flow paths based on few sensory data.

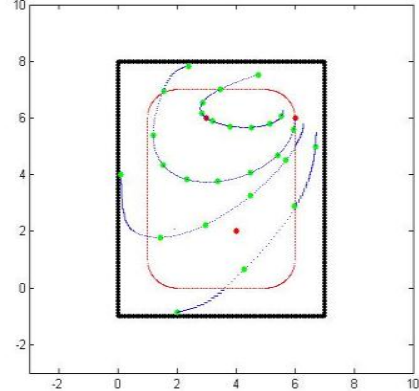

Figure 13. Boundary confined air-borne particle paths going through two sensors in a rectangular room

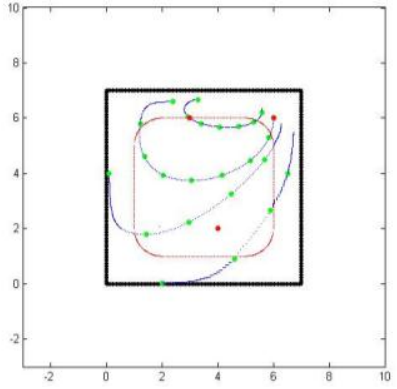

Figure 14. Boundary confined air-borne particle paths going through two sensors with twice the air speed

\section{COMPARE AND VALIDATE OUR APPROACH}

To compare and validate our approach, we use exact analytical methods for simpler cases and use finite-element method based business software (such as COMSOL) for more complicated cases.

The analysis of airborne particle motion is identical to the fluid motion analysis in physics. The fluid motion is governed by the Navier-Stokes nonlinear partial differential equation [6], such that motion in the two dimensional space satisfies:

$$
\begin{aligned}
& \frac{\partial u}{\partial x}+\frac{\partial v}{\partial y}=0 \\
& u \frac{\partial u}{\partial x}+v \frac{\partial u}{\partial y}=-\frac{1}{\rho} \frac{\partial P}{\partial x}+v\left(\frac{\partial^{2} u}{\partial x^{2}}+\frac{\partial^{2} u}{\partial y^{2}}\right) \\
& u \frac{\partial v}{\partial x}+v \frac{\partial v}{\partial y}=-\frac{1}{\rho} \frac{\partial P}{\partial y}+v\left(\frac{\partial^{2} v}{\partial x^{2}}+\frac{\partial^{2} v}{\partial y^{2}}\right)
\end{aligned}
$$


Where $u$ and $v$ are the components of the velocity in the $x$ and $y$ directions, $\rho$ is the fluid density, and $P$ is the pressure.

The analytical solutions to the Navier-Stokes equations depend on the initial and the boundary conditions, and the exact solutions exist only for simple cases.

In this paper, we will assume that the particle flow dynamics is two dimensional, and it's uncompressible, inviscid, and irrotational. If $\mathrm{D}$ is a simply connected domain in $(\mathrm{x}, \mathrm{y})$ and the flow is irrotational, the integral $\int u d x+v d y$ is independent of path in D. If we integrate from a fixed point $(a, b)$ to a variable point $(\mathrm{m}, \mathrm{n})$, then the integral becomes a functions of the point $(m, n)$ :

$$
\Phi(\mathrm{m}, \mathrm{n})=\int_{(a, b)}^{(\mathrm{m}, \mathrm{n})}(u d x+v d y)
$$

The function $\Phi(x, y)$ is called velocity potential of the motion. Since the integral is independent of path, $u d x+v d y$ is an exact differential, namely, the differential of function $\Phi(x, y)$, that is,

$$
u d x+v d y=\frac{\partial \Phi}{\partial x} d x+\frac{\partial \Phi}{\partial y} d y
$$

Form (3),

$$
u=\frac{\partial \Phi}{\partial x}, \quad v=\frac{\partial \Phi}{\partial y}
$$

By substituting $\mathrm{u}$ and $\mathrm{v}$ in (4), into (1) we see that $\Phi(x, \mathrm{y})$ satisfies Laplace's equation:

$$
\nabla^{2} \Phi=\frac{\partial^{2} \Phi}{\partial x^{2}}+\frac{\partial^{2} \Phi}{\partial y^{2}}=\mathbf{O}
$$

By the above theoretical derivation, we use Laplace's equation to model fluid motion.

Let $\Psi(x, y)$ be a conjugate function of $\Phi(x, y)$. The function $\Psi(x, y)$ is called the stream function of the flow. The curves $\Psi(x, y)=$ const are the streamlines of the fluid. We know that both $\Psi(x, y)$ and $\Phi(x, y)$ have continuous second derivatives as shown in [7]. Then the complex function

$$
F(\mathrm{x}, \mathrm{y})=\Phi(x, \mathrm{y})+i \Psi(x, \mathrm{y})
$$

is analytic in the region of the flow. This function is called the complex potential of the flow.

The velocity of the flow can be obtained by differentiating (6) and using Cauchy-Riemann equations [8]; we find

$$
F_{x}(\mathrm{x}, \mathrm{y})=\frac{\partial \Phi}{\partial x}+i \frac{\partial \Psi}{\partial x}=\frac{\partial \Phi}{\partial x}-i \frac{\partial \Phi}{\partial y}=u-i v
$$

General solution to (7) can be complicated and unnecessary for the simple cases that we are considering. Indeed, we will assume the function $\mathrm{F}$ from the initial flow and determine the specifies by substituting the functions into the differential equations. Because of the uniqueness of the solution to the differential equation under initial and boundary conditions, if the function F satisfies (5) and the boundary conditions, then it's the unique solution. In a case, Inviscid flow past a cylindrical obstacle.

In this case, the complex potential for an ideal fluid flowing from left to right across the complex plane and around the unit circle $|z|=x^{2}+y^{2}=1$.

We use the fact that the conformal mapping $w=S(z)=z+\frac{1}{z}$ maps the domain $D=\{\mathrm{z}:|z|<1\}$ one-to-one and onto the $w$ plane slit along the segment $-2 \leq u \leq 2, v=0$. The complex 
potential for a uniform horizontal flow parallel to this slit in the $w$ plane is $F_{1}(w)=A w$, where A is a positive real number. The stream function for the flow in the $w$ plane is $\psi(\mathrm{u}, v)=A v$ so that the slit lies along the streamline $\psi(x, y)=0$.

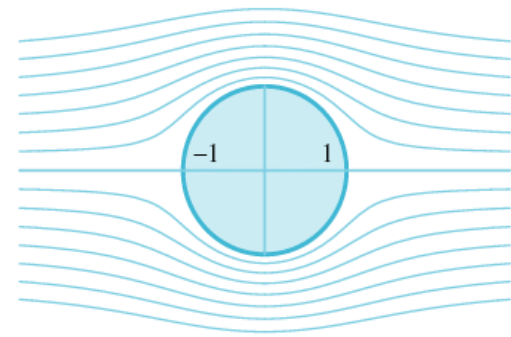

Figure 15. Fluid flow around a circle

The composite function $F_{2}(z)=F_{1}(\mathrm{~S}(\mathrm{z}))$ determines the fluid flow in the domain $\mathrm{D}$, where the complex potential is $F_{2}(z)=F_{1}(\mathrm{~S}(\mathrm{z}))=\mathrm{A}\left(\mathrm{z}+\frac{1}{z}\right)$, where $A>0$. We can use polar coordinates to express $F_{2}(z)$ as

$$
\begin{aligned}
& F_{2}(z)=F_{2}\left(r e^{i \theta}\right)=\mathrm{A}\left(r e^{i \theta}+\frac{1}{r e^{i \theta}}\right) \\
& =A\left(\mathrm{r} \cos \theta+i r \sin \theta+\frac{1}{r \cos \theta+i r \sin \theta}\right) \\
& =A\left(\mathrm{r} \cos \theta+i r \sin \theta+\frac{r(\cos \theta-i r \sin \theta)}{r^{2}}\right) \\
& =\mathrm{A}\left(\mathrm{r}+\frac{1}{r}\right) \cos \theta+i A\left(\mathrm{r}-\frac{1}{r}\right) \sin \theta
\end{aligned}
$$

The streamline $\quad \psi(r, \theta)=A\left(r-\frac{1}{r}\right) \sin \theta=0 \quad$ consists of the rays $r>1, \theta=0$ and $r>1, \theta=\pi$ along the $\mathrm{x}$ axis and the curve $r-\frac{1}{r}=0$, which is the unit circle $|z|=r=1$. Thus the unit circle can be considered as a boundary curve for the fluid flow.

The approximation $F_{2}(z)=F_{1}(\mathrm{~S}(\mathrm{z}))=\mathrm{A}\left(\mathrm{z}+\frac{1}{z}\right) \approx \mathrm{Az}$ is valid for large values of $\mathrm{z}$, so we can approximate the flow with a uniform horizontal flow having speed $|V(x, y)|=A$ at points that are distant from the origin.

Picking arbitrary two points in the $x-y$ plane $\left(\left(x_{1}, y_{1}\right)\right.$ and $\left.\left(x_{2}, y_{2}\right)\right)$ be the two sensor's locations, we use the interpolation method can get the fluid propagation path between the two points. However, after considering the chemical concentration, the endpoints of the path should be modified. When the two points are chosen on a same streamline, the streamline can be calculated to $y=a x^{3}+b x^{2}+c x+d$. The error term is $\int_{\left(x_{1}, y_{1}\right)}^{\left(x_{2}, y_{2}\right)}\left(\frac{k}{A x}-a x^{3}-b x^{2}-c x-d\right)^{2} d x$, where the $\mathrm{A}$ is a positive real number and (a,b,c,d) are four parameters that is related with the coordinates of the two points and derivatives of the two points .

The below figure give the error curve between fluid dynamic method and our proposed method. The left one is fluid path plot using our proposed method, the right one is the error curve. For a certain path, we select this red path be the considered path, then fix one point $x_{1}=1$, change $x_{2}$ in the region $(0, \infty)$. In the figure 16, the error curve shows the error value of fluid path derived by our proposed method. 

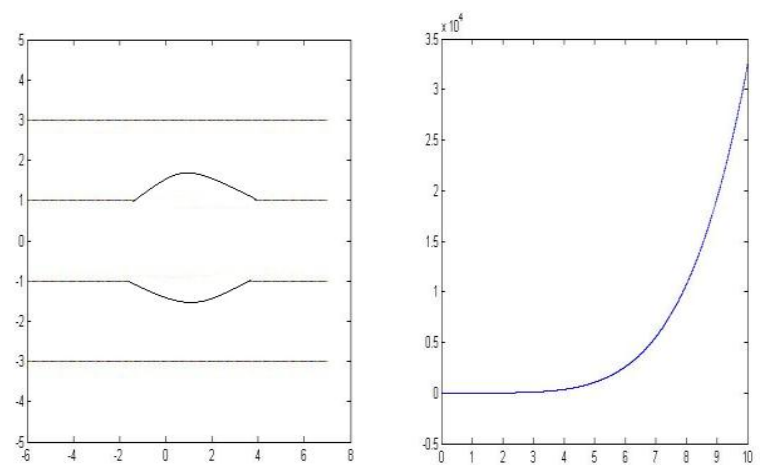

Figure 16. The performance of interpolation and extrapolation for the fluid flow around infinite wall

In summary, based on all of the above results, they can conclude that the error term is related with the coordinates and derivatives of two sensors and the boundary condition. Obviously, it's coincident with the natural law. The closer distance of two sensors, the error term of the chemical propagation path is smaller. In the other words, the more sensors we use, the better result we get, since the distance of any two sensors become closer. As for the boundary condition, the simplest condition is free boundary in which the error is equal to zero. It's because the cubic hermit Spline function can approximate any function, however, the path on the complex boundary will bring higher order error term. When the order of Spline function is infinite, the error of approximation should be zero.

\section{The framework and algorithm of mobile robot control architecture \\ 4.1. Odor Sensor \& Anemometer sensor}

The important parts of mobile robot for detecting and tracking chemical source are odor sensors and anemometer sensors. The odor distribution changes rapidly in dynamic advection-diffusion airflow environments. Therefore, odor cannot be intercepted in most workspaces, and even when the robot intercepts the odor, the interception time is brief because of the rapidly changing plume [9]. In such a case, the robot needs to respond immediately to the presence of the odor arrival. The odor sensor detects the presence of an airborne substance through a change in the electrical resistance of a chemically sensitive carbon-doped polymer film [10]. It is well known that both the response time and the recovery time of the commonly used metal-oxide-semiconductor gas sensors are lengthy (of the order of several seconds to tens of seconds). Therefore, with a fixed concentration threshold in natural outdoor airflow environments, both the odor detection and no detection events can lag significantly. The larger the fixed threshold, the further the odordetection event lags behind the real odor interception, and the bigger the risk of not detecting the odor contact (for example, if the real odor concentration is $20 \mathrm{ppm}$, with the fixed threshold of $25 \mathrm{ppm}$, the robot fails to find the odor plume). The smaller the fixed threshold, the greater the chance of invalid detection events (i.e., even though the gas sensor is not in the odor plume, odor detection events still occur). To make the robot quickly and reliably respond to odor interception, a binary concentration with an adaptive threshold rather than a fixed threshold is used in this research. The adaptive threshold used in this research was proposed in [11] and can be represented as:

$$
\bar{S}_{K}=\left\{\begin{array}{lr}
\lambda \bar{S}_{K-1}+(1-\lambda) \mathrm{S}_{K} & K \geq 1 \\
\mathrm{~S}_{K} & K=0
\end{array}\right.
$$

Where $\mathrm{S}_{K}$ is the measured odor concentration at time step k, $S_{0}$ is the initial concentration detected at the start and $\lambda \in[0,1]$.The binary concentration with adaptive threshold is defined as:

$$
z_{k}=\left\{\begin{array}{lr}
1 & S_{K}-\bar{S}_{K-1}>0 \\
0 & \text { else }
\end{array}\right.
$$

Where $z_{k}$ indicates an odor detection event at time step $\mathrm{k}$.

An anemometer mounted on the robot can provide relative velocity between the airflow and the anemometer. The air- flow velocity described in this paper was a modified value, with the velocity of the robot's motion being subtracted from the anemometer measurement. The anemometer is a Shibaura F6201-1 air flow sensor, which can sense wind flow down to $0.05 \mathrm{~m} / \mathrm{s}$. It is enclosed in a tube to provide 
unidirectional sensitivity, which, combined with a scanning behavior, allows the robot to measure wind direction. When wind direction information is required, the robot first rotates 90, and then rotates slowly 360 while reading the wind sensor output and finally rotates back to the heading corresponding to the highest sensor value.

\subsection{Mobile robot control architecture}

In this paper, we proposed a novel control algorithm of mobile robot for detecting and tracking chemical source.. At each time step, the location of the odor source is estimated and the termination condition is checked. If the terminated condition is satisfied, the mission of detecting chemical source is ended, otherwise the mobile robot move more locations to do more measurements and updates the gas distribution map and odor source localization.

This tracing strategy is described as follows. At first, the robot moves arbitral at two locations, because the chemical particle flow is derived by at least two sensor nodes. After the first step, the robot can know the source location roughly by the gas distribution map. Then, the robot moves to a new location where it is along the direction of chemical particle propagation. When we got a new location and updated the gas distribution map, we have two methods. We can use all of the sensors to map the chemical particle propagation, or we can use new sensor to update the map derived by the former sensors. The robot motion step can be adaptive through the real-time the gas distribution map.

\section{Experimental Result}

From the Google Earth, a real map of Missouri University of science and technology can be derived. Using the edge detection method, the processed map only exits the main buildings. Figure 17 showed the real map captured from Google Earth. Figure 18 shows the real map processed by the edge detection approach.

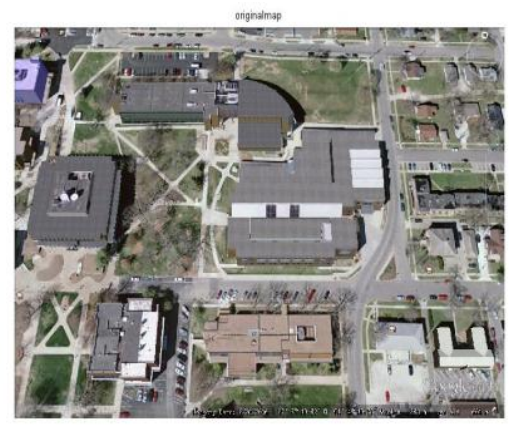

Figure 17. A real map of Missouri University of science and technology from the Google Earth

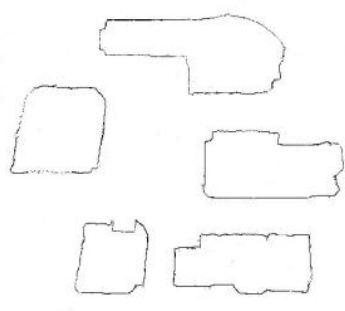

Figure 18. A real map of Missouri University of science and technology processed by edge detection method

Using all of above approaches, we can extend the path unlimitedly. However, in fact the entire paths are not completely exact. We should know where we can start a path and where the path ends to decide the effective range of the paths. The following assumption is introduced for the paths' credible section.

Assumption 8: Based on the measurement range of sensors, only the sections of the paths where are surrounded by sensors are credible.

Because the information collected by the sensors is local variable, only the data on the sensor location is completely correct and the entire paths are constructed based on the sensors' data. The section of the paths where are near the sensors have more credible level, while the sections of the paths where are far from the sensors should have a low credible level. Using all sensors be the vertices, we can plot a convex polygon in which we believe the paths are completely correct. Keeping an equal distance with the polygon, we get a zone which is encircled. In this encircled section, the paths are correct in certain probability. In the Figure 19, the green dotted lines show the exact zone, the yellow dotted lines designate the uncertain region. At the same time, the boundary lines of uncertain zone truncate the particle paths. Hence, we are able to obtain both the starting points and ending points of the paths. Beyond this uncertain zone, the path is not thought be credible. It's because the portions beyond the uncertain zone are derived by extrapolation without any updated data from nearby sensors. 

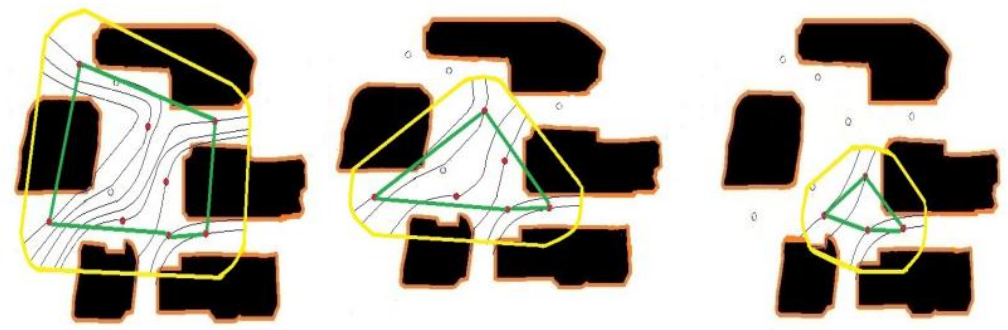

Figure 19. The credible sections of chemical path using different number of working sensor

To verify the effectiveness of the proposed assumptions, real simulation is developed by COMSOL software that is used to analyze complex flow of fluid dynamics. We assume that the wind is from southwest 45 degrees and the configuration is on same size and position with the Google map. This software is accorded to Finite Element method to process the fluid dynamic issue, so we want to use the result from this software to do a comparison. From the results produced COMSOL, we are able to obtain the velocity of airflow at every point and the particle paths. We select ten points, and record its locations and velocities on these points. Figure 20 shows the steam line of airflow propagation which illustrates the chemical particles dispersion.

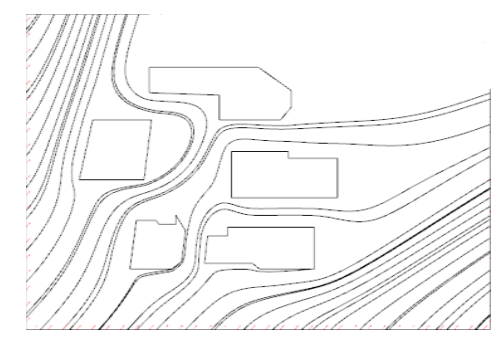

Figure 20. Air-borne particle paths going through ten sensors in a real map processed by COMSOL

If the results using our proposed approach and the results using COMSOL are identical, it can support our proposed approach is correct. Our approach uses the same map, locations and velocities of sensors that are selected from the result of COMSOL. The circles signify the locations where we fixed sensors. The red circles mean the sensors are working, and the blank circles mean the sensors are not working. Comparing the figure 19 and figure 20, we can find the airflow path is almost same. Figure 19 show the cases where different number of working sensors existed. When the working sensors are changed from eight to one, the maps of the airflows are different. A larger number of working sensor produce results closer to the real data obtained from COMSOL. The sensor's dispatch affects the result of simulation also. It is obvious that optimized dispatch can collect more information for the system. For an extreme example, if we fix all of the sensors in a small area together, the effective paths will existed in this small area only.

\section{CONCLUSION}

There are many useful and humanitarian applications that can locate the source of a chemical source. Currently, the majority of work in this area uses reactive control schemes that track an odor plume along its entire length, which is slow and difficult in cluttered environments. This paper employs a high-level control scheme. The interpolation and extrapolation method is used to model the particle path in the sensors' environment. Then a reasoning system use the path model to get the velocity, chemical concentration at any point on the map and predict the most probable locations of the odor source. This approach has been shown to be effective for odor localization in a known environment, without the need for the robot to travel to the source.

With the further development there is great potential for this approach to lead to many valuable applications by generalization to a wider range of environmental configurations. The paper present development to solve the problem there exist obstacles and opening in the environment. The approach gives the mode of the particle path surrounding the obstacles and openings. The development has successfully applied in general environment, because the propagation of the chemical particle can go through obstacles 
and opening in actual case. In addition, this paper is the first example of using interpolation and extrapolation method to model the particle path that applied in a real environment.

In addition, the result of simulating the particle path in the real map of campus is approximate with the result from the fluid dynamic analysis software, COMSOL. Hence, this approach can approximately map the particle path in real cases. Future work will concentrate on two areas of development: development of more general particle path modeling algorithm, and implementation of a range data acquisition system to enable autonomous map the building.

\section{REFERENCES}

[1] H. Poor, An Introduction to Signal Detection and Estimation. New York: Springer-Verlag, 1985, ch. 4.

[2] P. Hayes, "The second naive physics manifesto," in Formal theories of the commonsense world (J. Hobbs and R. Moore, eds.), pp. 1-36, Norwood, New Jersey: Ablex Publishing Corporation, 1985.

[3] G. Kowadlo and R. Russell, "Naive physics for effective odor localisation," in Australasian Conference on Robotics and Automation, (Brisbane, Australia), 2003.

[4] G. Kowadlo and R. Russell, "Advanced air ow modelling using naive physics for odour localisation," in Australasian Conference on Robotics and Automation, (Sydney, Australia), 2005.

[5] G. Kowadlo and R. Russell, "Using naive physics for odor localization in a cluttered indoor environment," Autonomous Robots, vol. 20, no. 3, pp. 215-230, 2006.

[6] G. Kowadlo and R. Russell, "Improving the robustness of naive physics airow mapping, using bayesian reasoning on a multiple hypothesis tree," in IEEE International Conference on Robotics and Biomimetics, (Kunming, China), 2006.

[7] G. Kowadlo, D. Rawlinson, R. Russell, and R. Jarvis, "Bi-modal search using complementary sensing (olfaction/vision) for odour source localisation," in IEEE International Conference on Robotics and Automation, (Orlando, Florida), 2006.

[8] H. Ishida, "Study of autonomous mobile sensing system for localization of odor source using gas sensors and anemometric sensors," Sensors \& Actuators, vol. 45, no. 2, pp. 153-157, 1994.

[9] T. Duckett, M. Axelsson, and A. Saffiotti, "Learning to locate an odour source with a mobile robot," in IEEE International Conference on Robotics and Automation, 2001.13

[10] Jigong Li, Qinghao, Meng, "Odor source localization using mobile robot in outdoor airflow environments with a particle filter algorithm,” Autonomous Robot. April 2011, Volume 30, Issue 3, pp 281-292.

[11] A.T.Haves, "Distributed odour source localization", IEEE Sensors Journal (Volume 2, Issue 3), June 2002.

[12] E. H. Miller, "A note on reflector arrays (Periodical style-Accepted for publication)," IEEE Trans. Antennas Propagat., to be published.

[13] C. J. Kaufman, Rocky Mountain Research Lab., Boulder, CO, private communication, May 1995.

[14] J. U. Duncombe, "Infrared navigation-Part I: An assessment of feasibility (Periodical style)," IEEE Trans. Electron Devices, vol. ED-11, pp. 34-39, Jan. 1959.

\section{BIOGRAPHIES OF AUTHORS}

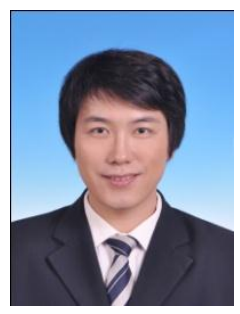

XIANG GAO, PhD candidate in electrical and computer engineering, Missouri University of Science and Technology. Research interests are: Control system design, wireless sensors network, navigation system, mobile robot.

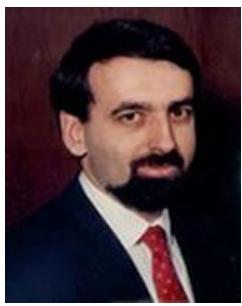

LEVENT ACAR, Associate Professor, electrical and computer engineering, Missouri University of Science and Technology. Research interests are: intelligent control of functional systems, neural networks applied to control, hierarchical design and control of large-scale systems, optimal and suboptimal control for interconnected systems, distributed computational methods of optimal control strategies. 